\title{
Robosoccer-RU Open Simulation League: Principles and Algorithms
}

\author{
D.E. Okhotsimsky ${ }^{1}$, V.E. Pavlovsky ${ }^{1}$, A.N. Touganov ${ }^{1}$, A.G. Plakhov ${ }^{1}$, \\ V.V. Pavlovsky ${ }^{1}$, S.S. Stepanov ${ }^{2}$, and A.Yu. Zaslavsky ${ }^{2}$ \\ 1 Keldysh Institute of Applied Mathematics of RAS (KIAM) \\ Moscow State University, Moscow, Russia \\ vlpavl@spp.keldysh.ru \\ 2 N-th.com Research, Dnepropetrovsk, Ukraine \\ robocup@n-th.com \\ http://n-th.com/
}

\begin{abstract}
In this paper we outline basic principles and competition rules used in a Computer soccer Simulation contest held in Eastern European countries (Robosoccer-RU League). The programming environment of this tournament ("Virtual Soccer" Software package) is described, as well as base algorithms that are implemented for powering team agents. A comparison is given between the reviewed approach and the one used in the RoboCup Simulation League, and directions for future convergence are drafted.
\end{abstract}

\section{Introduction}

Last decade has revealed an increasing interest within the computer science community to the research into autonomous intelligent agents and adjacent fields. These investigations cover a wide spectrum of fields, including Distributed Artificial Intelligence Systems, Artificial Life, Distributed Problem Solution and a number of others. One of the most appropriate test-benches for the relevant methods and algorithms is the computer simulation of the game of soccer. The RoboCup Simulation League has been holding the worldwide computer soccer tournaments for several years [1-7].

A closely related initiative was started in 2001 in Eastern European countries (mainly the former republics of the Soviet Union), when the first competition within the so called Robosoccer-RU League was organized. In this paper, we review basic principles and rules that underlie this contest, and describe in some details the game models, programming environment and algorithms used for autonomous software agents. A qualitative comparison with the RoboCup simulator is given, the design of the present implementation does not allow to carry out a direct match between correponding algorithms.

\section{The Basic Principles of Robosoccer-RU Simulation}

The main principle of the simulation model used by the Robosoccer-RU computer soccer League is the priority of the core algorithms forming the base for 
intelligent behavior of an agent. To put it differently, all the technical aspects related to agent-server interaction, obtaining of visual information by agent, and similar tasks, are simplified to the maximal extent. Therefore, the teams that take part in the competition are able to focus their efforts directly on developing the optimal strategy for players in the multi-agent environment.

Another significant difference is that in Robosoccer-RU League this is the strategy of a team of robots that is the object of research. This approach imposes serious restrictions upon several of the key parameters of the simulation system, and the Robosoccer-RU server cannot thus be considered as a simplified version of the model used in the RoboCup. Among other things, the number of players per team can vary and currently the typical values lie within the $3-5$ range.

The following list enumerates the most important differences between the RoboCup [1] and Robosoccer-RU simulation models:

1. All the data from server is sent to client in structured binary form, which makes the parsing of textual messages unnecessary.

2. Full information about all movable objects (i.e. their positions and velocities) is known at any moment, up to an error communicated by the server. Thus, there is no need to analyze the visual information based on flags, lines and other markers. Consequently, players have no heads and the notion of a visible angle is not introduced.

3. There is no delay between receiving information and deciding on the next move. At every simulation step the agent possesses the current information (full or with a certain error), which it uses to decide on what changes to apply to its state.

4. The notion of stamina is not introduced.

The Robosoccer-RU competitions are carried out in real-time. There is, however, a possibility of running the server at an increased speed (limited only by the power of underlying hardware). Therefore, a very effective way exists to gather vast amounts of data for testing and optimizing the game algorithms.

\section{The Physical Model and Rules of the Game}

The game is played, alike RoboCup [1], in two dimensions. All the objects on the field are round and have predefined sizes and masses. A player is 2.5 times as big as the ball, and consequently six times heavier. Interactions between them are governed by a quite realistic physical model, which describes the collisions as quasi-inelastic with dissipation of energy. The degree of inelasticity is controlled by a configurable parameter. Currently, the value of this parameter corresponds to almost elastic collision; the reason for such choice is the fact that the players hit the ball with their bodies.

There are two controlling parameters that allow player to change its state: linear acceleration $d V$ and angular velocity of player's rotation $d \alpha$ (corresponding to the Robocup's commands dash and turn). To receive information, agent used the set of functions provided by the SDK. For instance, the function GetX (short 
ObjectNr) returns the value of $x$-coordinate of the object with the number ObjectNr, GetdY (short ObjectNr) returns the $y$-component of its velocity, and so on. Upon gathering all the necessary information, player sets new values for the controlling variables $(d V, d \alpha)$. There are, of course, certain limits imposed on the values of the acceleration and velocity, so that players' motion is inertial.

At present, there is no dedicated goalie in the team; every player can defend the goal, but it has no special means (like catch command) for this purpose.

The rules of the game are also simplified. In their today's form they correspond rather to the rules of mini-football (futsal). All the objects (players and ball) reflect from the edges of the field, that is corners, off-sides, outs and other standard situations are absent from the game.

\section{The "Virtual Soccer" Software Package}

The game simulation model is as follows [8]. The programming environment was developed for different platforms, including the MS Windows OS; all those programs implement identical mechanical models, while the algorithms controlling the players' behavior are realized as loadable modules and can be changed. All the implementations are compatible on the configuration file level, and use the same mechanism for loading team modules. The main goal of the simulation is optimization of the algorithm parameters and selection of the most effective one. Algorithms of the competing teams can belong to the same class, or to different ones.

The simulation program is composed of the three principal parts, the server and two loadable modules, modeling the behavior of the two teams. The server contains the core of the system and glues all the modules together. The implementation of loading varies from platform to platform, under Windows the DLL protocol is used. The game process is modeled using timer ticks; the simulator steps can correspond to the real (astronomic) time, or their length can be decreased and the game will be played at a high speed.

At every simulator step the server calls the team modules, Team1 and Team2 in that order. Every team module is called so many times per one step, as many players per team were defined (this number may vary, as noted above). The team module must return to the server the controlling parameters for every player in the team. While performing the calculation of the parameters, the module can access the API-functions of the server and receive the current state (position and velocity) of any object on the field. In this way the visual input of information into the system controlling the players is implemented. At the end of simulation step the server calculates next positions and velocities for all objects using the commands received from teams and internal mechanical model. Dynamics of the players' and ball's motion and their collisions are modeled. The current state of the game is determined (including goal and other situations), and if necessary, the server transfers the game into one of the predefined states, e.g. into state "before kick-off" after a goal is scored. 
The present version of the server software implements a model of the game with complete information, when above the field a host-camera is placed that monitors all the events on the ground (or one such camera for each team, with identical information), and the players are controlled by two host-computers. However, if need be, it is possible for team modules to model an autonomous control system of a separate player.

A screen-shot of a match played within the "Virtual Soccer" simulation environment is shown in Fig. 1. This figure also illustrates some elements of the program interface.

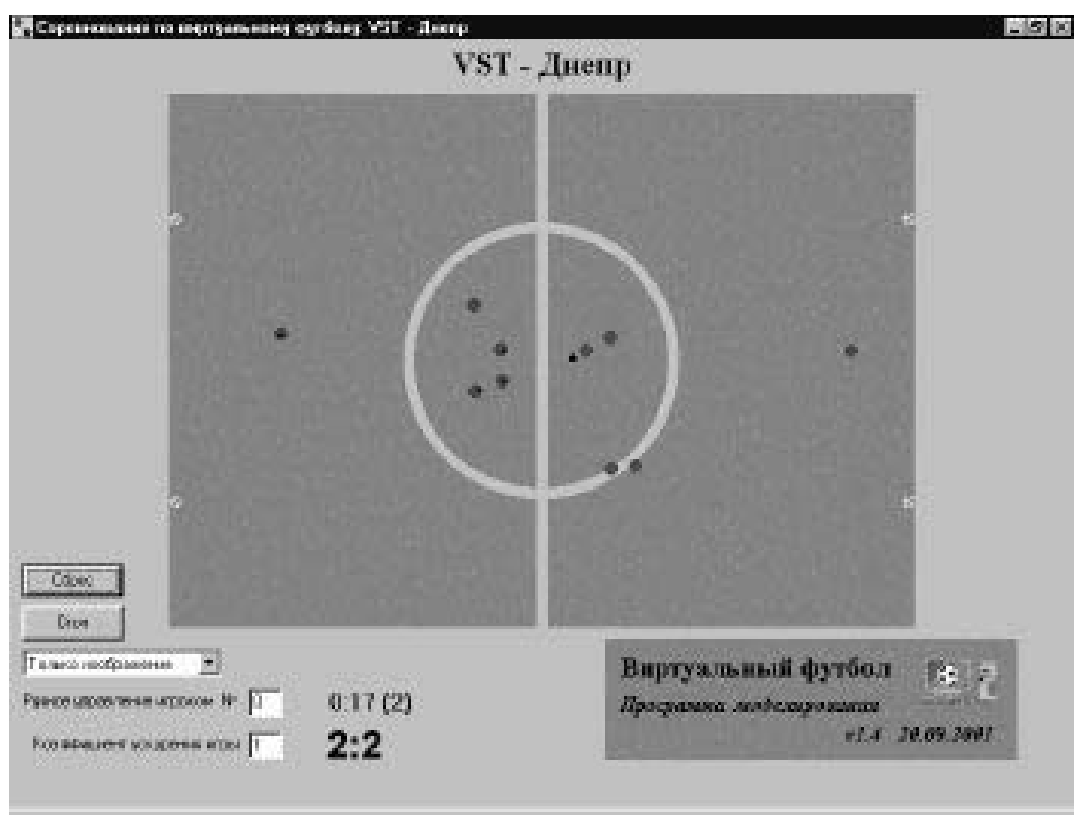

Fig. 1. A screen-shot of the soccer match played in "Virtual Soccer" simulation package.

As was mentioned above, several versions of the modeling server and tools for developing team modules are available for different computer platforms. Server can be run under operating systems MS DOS, MS Windows and Linux, and seven most widely used application development environments are supported for such languages as $\mathrm{C} / \mathrm{C}++$ and Pascal (for DOS and Windows) and GNU $\mathrm{C} / \mathrm{C}++$ compiler (for Linux).

\section{An Example of Algorithm for Team Control}

In the course of performed experiments a number of heuristic algorithms for controlling soccer-playing robots were investigated [9-11]. Here we outline, as 
an example, the algorithm developed by the team of the Keldysh Institute of Applied Mathematics of RAS (KIAM) for the Robosoccer-RU simulator, and used during the latest championship.

These algorithms, while differing by the values of characteristic parameters and the methods by which the control functions are calculated, all belong to the same class which can be defined as follows. Every player is assigned a rectangular area on the field (zone of responsibility), which can possibly be the whole field. The player must remain within the boundaries of this zone, with the only exception being that it can leave it as a result of inertial motion. In the latter case player should try to return to its zone as soon as possible.

At every simulation cycle player determines the current aim-point on the field, i.e. the spot it is moving to. We will call these spots special; the special points of a player are plotted in Fig. 2 .

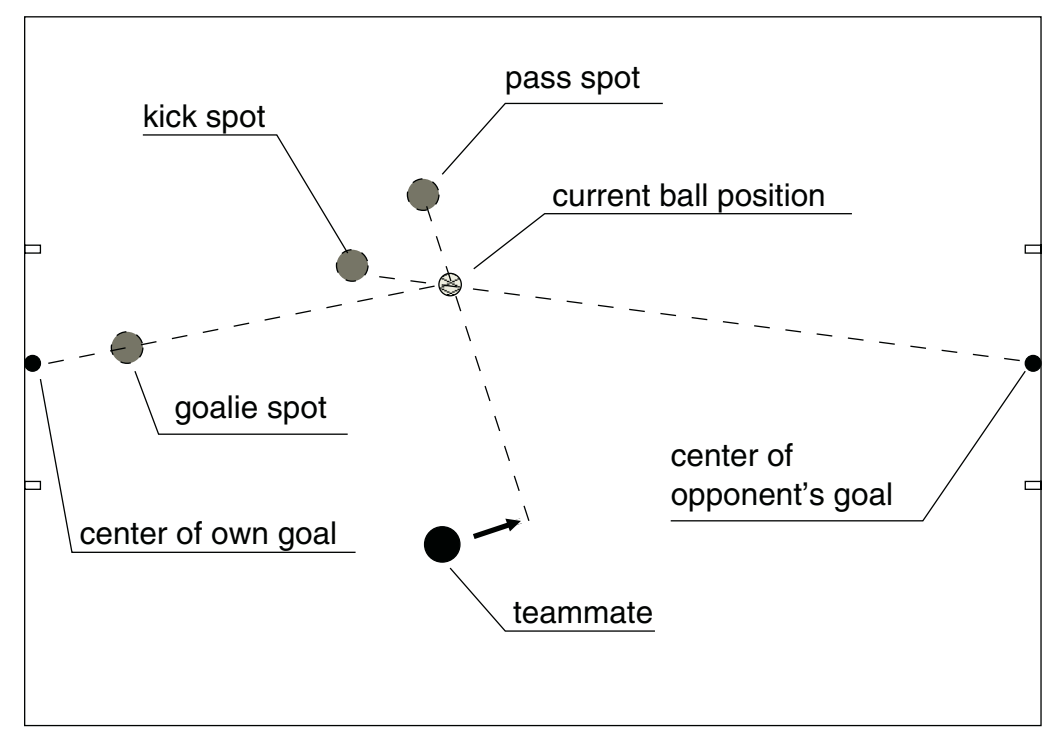

Fig. 2. The special spots (aim points) of a player are plotted, which are used for making decision on the next action.

There are several kinds of special points, namely the kick spot (from which it is possible to score a goal), the goalie spot (moving there player would defend the own goal) and the pass spot. Positions of all these points depend on the complete information about positions and velocities of all players and ball, and change quickly with time.

Let us now describe the geometric properties of the special points. The kick spot lies on the continuation of the ray extending from the center of the opponent's goal through the center of the ball. The distance from this point to the ball is one of the adjustment parameters of the algorithm. The goalie spot is the 
point on the line joining the center of the own goal with the ball. In different algorithms, either the distance player-goal, or the distance player-ball, are used as parameters. In the latter case, the responsibility zone for the goalie is chosen close to the goal to ensure that the goalkeeper does not follow the ball too far from the goal. Finally, the pass spot lies on the line joining the forecast position of the teammate (the point of interception where it would meet the ball after pass) with the ball's position.

Every algorithm is characterized by a set of parameters, which determine the priorities of different special points. The special points for all players are calculated by the team controlling module by means of position forecast functions for the objects on the field. Agent chooses the current aim among the calculated special points differently for different algorithms: by minimizing the time to reach the spot (algorithms with short forecast), by maximizing the strategic game effect (algorithms with full forecast), or by choosing the one with the highest priority (algorithms with priorities as adjustable parameters).

All the algorithms that were used can be broken into three main classes. The first one ("hard", or deterministic, algorithms) use only the special spots scheme outlined above. The second class (extended algorithms) adds models of arrangement of players on the opponent's half, as well as a tactical task (defense) that sets high priority to kicking the ball out of the own half-field. One more special point is introduced, similar to the kick spot, but with the distance player-ball shorter than the radius of a player. This allowed to realize effective dribbling, and at the same time block the kicks toward the own goal. The third class includes models where the players are assigned certain roles (goalkeeper, forward, defenseman, midfielder, etc), and allowing the players to change the role based upon the current situation on the field. These role algorithms were chosen as the base for carrying out the first tournament using the simulation package "Virtual Soccer".

To sum all this up, the parameters of the reviewed model are: the characteristics of robots, their responsibility zones, forecast depth and the ways used to pick it, geometrical parameters and priorities of special points, class of algorithm, and methods of assigning roles to players. These parameters were optimized by means of repeatedly running the game at increased speed. In the course of the research the "machine evolution" method was used among others, and best-playing teams were selected and optimal values of the parameters (and sets of parameters) were determined. In addition, the programs controlling goalie agents were "trained" separately, for in the real game there was not enough work for the goalkeepers to reliably test relevant algorithms.

As a separate stage of the investigation, a number of experiments were conducted where some of the agents were controlled by a human operator. The main aim was to obtain an extra measure of the model effectiveness. It was shown that fully automatic teams as a rule win the matches against human-aided sides. 


\section{Short History and Future Directions}

At the conference Artificial Intelligence '2000 held in Katsyvely (Crimea, Ukraine) a group of researches representing the Keldysh Institute of Applied Mathematics of RAS and Moscow State University (Russia) put forward the idea to organize a competition similar to the RoboCup, but with simplified rules oriented to facilitate soccer robots simulation and control. Simulation software was developed, including the server and SDK for team modules programming, for operating systems MS Windows and Linux. In October, 2001 at the conference Artificial Intelligence '2001, the preliminary matches were played by the designed rules.

In December of 2001 within the framework of the annual scientific Workshop and Festival Mobile Robots'2001 held in Moscow University, the first official championship of the Robosoccer-RU League took place. Among the participants there were 10 teams from Russia and Ukraine.

These competitions showed that the main principles used for designing the rules of the Robosoccer-RU League were well-grounded. The simplification of the technical tasks, including agent-server communication, allowed the participants of the project to concentrate their efforts on the important problems of controlling the agent's behavior and game strategy.

At present, a possibility is considered of modifying the rules used in Robosoccer-RU to make them more compatible with the RoboCup [1], while keeping intact the basic principles of information retrieval by the autonomous agents.

The following changes are suggested:

1. Increasing inelasticity of collisions between objects. This can be achieved by modifying the relevant parameter, while keeping the implemented dynamical model unchanged.

2. Introducing new control parameters allowing agents to kick ball with given power and in a given direction. Agents will thus have four commands available to them, corresponding to the RoboCup's commands dash, turn and kick. The boundary conditions (objects reflecting off the field edge) will most probably be left in place.

3. Introducing uncertainty into the information communicated by server to agents. To this end, all returned parameters will be accompanied by a standard deviation $\sigma$, which gives the estimate of possible error in the corresponding value. Therefore, agents will only know with a certain degree of confidence that the value of a parameter lies within some interval. For different objects and at different time the value of $\sigma$ can vary. It would grow for the objects that are out of the player's sight, i.e. situated behind or too far away from it. This would allow for a smooth transition from the model with complete information to the one with incomplete data, without adding the resource-consuming stage of visual information analysis. 


\section{Conclusion}

The Robosoccer-RU League is an open competition that can be attended by researchers from countries all over the world. At the present stage of its development, the main goal is to provide the test field for improving strategic algorithms for control and interaction between autonomous agents. The outcome of this rescarch can be applied to modeling complicated multi-agent environments, as well as used to enhance the core algorithms controlling the RoboCup Simulation League teams [1]. The results obtained so far during experiments and tournaments proved the effectiveness of the main modeling principles.

Further development of the simulation model is underway, which is intended to bring in more functional compatibility with the RoboCup Simulation League and with other RoboCup Leagues. Among other things, this will allow to directly compare algorithms developed within these two frameworks. The planned adjustments to the server program will allow to simulate the peculiarities of different RoboCup Leagues.

\section{References}

1. Mao Chen, Ehsan Foroughi, Fredrik Heintz, ZhanXiang Huang, Spiros Kapetanakis, Kostas Kostiadis, Johan Kummeneje, Itsuki Noda, Oliver Obst, Pat Riley, Timo Steffens, Yi Wang, and Xiang Yin: RoboCup SoccerServer L'ser Manual (http:/www.robocup.org/resource/ and links within).

2. Hiroaki Kitano, Yasuo Kuniyoshi, Itsuki Noda, Minoru Asada, Hitoshi Matsubara, and Ei-Ichi Osawa: Robocup: A challenge problem for AI. AI Magazine 18 (1997) $73-85$

3. I.Noda, H.Matsubara, K.Hiraki, I.Frank: Soccer Server: a tool for research on multiagent systerns. Appl. Artif. Intell. (AAI) Journal (USA) 12 (1998) 233250

4. Tonaka-Ishii Kumiko, Frank Ian, Arai Katsuto: Trying to understand RoboCup. AI Magazine 21 (2000) 19-24.

5. Manuela Veloso, Peter Stone, Kwun Han, and Sorin Achim: CMUnited: A team of robotic soccer agents collaborating in an adversarial environment. Proc. of the First Intcrnational Workshop on RoboCup, Nagoya, Japan, August, 1997.

6. Peter Stone and Mamela Veloso: A layered approach to learning client behaviours in the robocup soccer server. Appl. Artif. Intell. (AAI) Journal (LSA) 12 (1998) $165-188$.

7. Hugel Vincent, Bonnin Patrick, Blazevic Pierre: Using reactive and adaptive behaviours to play soccer. AI Magazine 21 (2000) 53-59.

8. D.E.Okhotsimsky, V.E.Pavlovsky, A.G.Plakhov, A.N.Touganov: Simulation of game of soccer robots and basic algorithms to control those robots. Artificial Intelligence (Ukrainian-Russian Journal) 3 (2000) 534540 (in Russian)

9. D.E.Okhotsimsky, V.E.Pavlovsky, A.G.Plakhov, A.N.Touganov: Towards the CLAWAR robots soccer playing - simulation of robotic soccer. Proc. of 4-th Int. Conf. on Climbing and Walking Robots CLAWAR'2001. Karlsruhe, Germany, 2426 September 2001, pp.451 456.

10. D.E.Okhotsimsky, V.E.Pavlovsky, A.G.Plakhov, A.N.Touganov, V.V.Pavlovsky: Simulation of game of soccer robots in the environment of 'Virtual Soccer' software package. Mechatronics, 2002. (in Russian, to be printed).

11. S.S. Stepanov, N.I. Stepanova: Turing-fifty years later. Artificial Intelligence (Ukrainian-Russian Journal) 3 (2000) 100-110 (in R.ussian). 